\section{MS44-P6 Measurement and calculation of thermal diffuse scattering of molecular \\ crystals: A case study of the \\ anti-tuberculosis drug pyrazinamide \\ Anders Østergaard Madsen ${ }^{1}$, Nanna Wahlberg ${ }^{1}$, Alexei Bosak ${ }^{2}$}

1. University of Copenhagen

2. European Synchrotron Radiation Facility

email: madsen@chem.ku.dk

Experimental information on the properties of phonons is traditionally obtained from inelastic neutron and inelastic X-ray scattering measurements. These experiments are time-consuming, and last at least a few days, and the information obtained gives only limited information about the phonon eigenvectors. The study of thermal diffuse scattering (TDS) offers a much faster route to obtain information about the low-frequency phonons, which has become feasible thanks to the advent of third generation synchrotrons in combination with large single-photon count detectors. The interpretation of TDS in the case of molecular crystals has been hampered by the lack of sufficiently accurate models of the lattice dynamics in crystals. Within recent years, it has become feasible to perform periodic DFT calculations of the lattice dynamics. Here, we demonstrate the calculation and analysis of TDS of pyrazinamide, a polymorphic tuberculosis drug. The polymorphs of pyrazinamide is an enantiotropic system, and the change in relative stability of the polymorphs with temperature is driven by differences in the acoustic phonons, which leads to differences in entropy. We examine the TDS patterns of these systems and discuss their features in relation to lattice-dynamical models derived from DFT calculations.

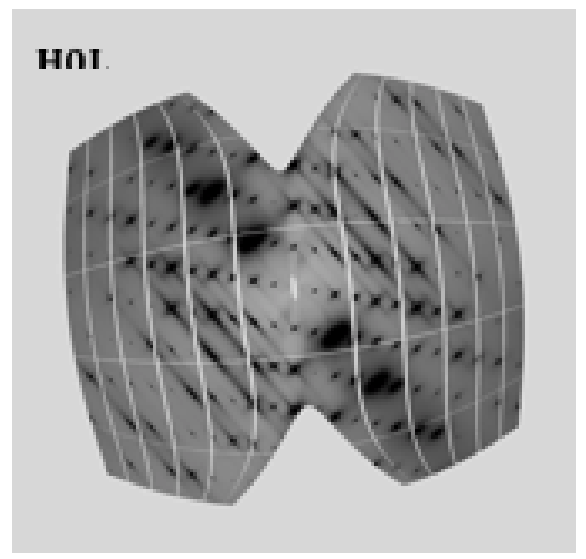

Figure 1. Results from a recent diffraction experiment on a pyrazinamide single crystal in the polymorphic delta-phase at room temperature showing a strong thermal diffuse scattering signal in the HOL plane.

Keywords: Thermal diffuse scattering, polymorphism, phonons
MS44-P7 X-ray diffraction studies of crystal structure and strain distribution in nanowires

Julian Stangl ${ }^{1}$

1. Johannes Kepler University Linz

email: julian.stangl@jku.at

Semiconductor nanowires exhibit a number of special properties making them interesting both for fundamental science as well as for different applications ranging from solar cells to fast transistors. In III-V semiconductors, the observation of hexagonal polytypes has spurred investigations of this crystal structure, which cannot be fabricated in bulk (at least not stable under ambient conditions). Some materials show a change in band alignment from indirect to direct fundamental band gap, such a behavior has also been predicted in the SiGe system. In this presentation, we will highlight the structural investigation of hexagonal $\mathrm{Si}$ material fabricated in nanowires, using high resolution $\mathrm{x}$-ray diffraction. Analysis of the diffuse x-ray diffraction patterns from the core-shell wires using finite element method yields the unit cell properties of hexagonal (lonsdaleite) silicon.
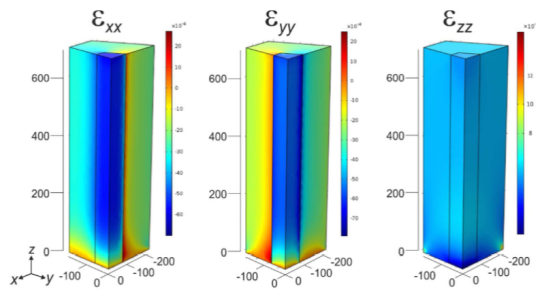

Figure 1. Finite element model simulations of the strain distribution in $\mathrm{GaP}$ core/ hexagonal Si shell nanowires.

Keywords: nanowires, hexagonal Si, diffuse scatering 